\title{
A New Compartment at Stereocilia Tips Defined by Spatial and Temporal Patterns of Myosin IIIa Expression
}

\author{
Mark E. Schneider, ${ }^{1 \star}$ Andréa C. Dosé, ${ }^{2 \star}$ Felipe T. Salles, ${ }^{1 \star}$ Weise Chang, ${ }^{1}$ Floyd L. Erickson, ${ }^{3}$ Beth Burnside, ${ }^{2}$ and \\ Bechara Kachar ${ }^{1}$ \\ ${ }^{1}$ Section on Structural Cell Biology, National Institute on Deafness and Other Communication Disorders, National Institutes of Health, Bethesda, Maryland \\ 20892, ${ }^{2}$ Department of Molecular and Cell Biology, University of California, Berkeley, California 94720, and ${ }^{3}$ Department of Biological Sciences, Salisbury \\ University, Salisbury, Maryland 21801
}

Class III myosins are motor proteins that contain an $\mathrm{N}$-terminal kinase domain and a C-terminal actin-binding domain. We show that myosin IIIa, which has been implicated in nonsyndromic progressive hearing loss, is localized at stereocilia tips. Myosin IIIa progressively accumulates during stereocilia maturation in a thimble-like pattern around the stereocilia tip, distinct from the cap-like localization of myosin XVa and the shaft localization of myosin Ic. Overexpression of deletion mutants for functional domains of green fluorescent protein (GFP)-myosin IIIa shows that the motor domain, but not the actin-binding tail domain, is required for stereocilia tip localization. Deletion of the kinase domain produces stereocilia elongation and bulging of the stereocilia tips. The thimble-like localization and the influence myosin IIIa has on stereocilia shape reveal a previously unrecognized molecular compartment at the distal end of stereocilia, the site of actin polymerization as well as operation of the mechanoelectrical transduction apparatus.

Key words: hair cells; myosin IIIa; stereocilia; hearing; myosin XVa; myosin Ic

\section{Introduction}

The staircase-shaped stereocilia bundle of the inner ear sensory hair cells detect mechanical stimuli by the activation of mechanoelectrical transduction (MET) channels at the stereocilia tips (for review, see Ricci et al., 2006). Stereocilia formation, hair bundle organization, and mechanosensory function depend on several unconventional myosins that when mutated cause stereocilia disruption and deafness. For example, myosin XVa is essential for stereocilia elongation (Belyantseva et al., 2005; Rzadzinska et al., 2005), myosin VIIa is required for maintaining proper cohesiveness of the stereocilia bundle (Boeda et al., 2002; Kros et al., 2002), and myosin VI is involved in maintaining the tapered base of the stereocilia (Seiler et al., 2004). Myosin Ic is another unconventional myosin that has been implicated in adaptation of the MET response (for review, see Gillespie and Cyr, 2004).

MYO3A, one of two human class III myosins (Dosé and Burnside, 2000), was shown to play a critical role in inner ear function because mutations in the motor domain of this gene lead to the progressive, nonsyndromic hearing loss DFNB30 (Walsh et al., 2002). Myosin IIIa is also expressed in the vertebrate retina (Dosé

Received July 1, 2006; revised Aug. 7, 2006; accepted Aug. 17, 2006

This work was supported by the National Institute on Deafness and Other Communication Disorders, Division of Intramural Research, National Institutes of Health. We thank Saeeda Latham and Patrick Riordan for technical help, Dr. Yuichiro Takada for the suggestion of using antigen retrieval techniques, and Drs. Aurea de Sousa and Ronald Petralia for comments on this manuscript.

*M.E.S., A.C.D., and F.T.S. contributed equally to this work.

Correspondence should be addressed to Dr. Bechara Kachar, Section on Structural Cell Biology, National Institute on Deafness and Other Communication Disorders, National Institutes of Health, Building 50, Room 4249, 50 South Drive, Bethesda, MD 20892-8027. E-mail: kacharb@nidcd.nih.gov.

DOI:10.1523/JNEUROSCI.2812-06.2006

Copyright $\odot 2006$ Society for Neuroscience ～0270-6474/06/2610243-10\$15.00/0 et al., 2003), and its Drosophila homolog, NINAC, is expressed in photoreceptors. NINAC interacts with actin filaments and with INAD, a PDZ (postsynaptic density-95/Discs large/zona occludens-1) scaffolding protein, and has been proposed to translocate and assemble proteins of the phototransduction signaling complex (Lee and Montell, 2004; Wes et al., 1999). Class III myosins share structural features with myosins XVa, VIIa, and Ic including an N-terminal canonical motor domain and a short regulatory neck domain containing a variable number of IQ motifs (Dosé and Burnside, 2000; for review, see Sellers, 2000). Myosin III proteins are unique in that they contain an $\mathrm{N}$-terminal kinase domain and undergo autophosphorylation ( $\mathrm{Ng}$ et al., 1996; Komaba et al., 2003). Deletion of the kinase domain does not prevent myosin IIIa localization to HeLa cell filopodia tips (Les Erickson et al., 2003), but this domain is essential for normal phototransduction in Drosophila (Porter and Montell, 1993). The myosin IIIa C terminus contains two conserved signature motifs termed myosin III tail homology domains, 3THDI and 3THDII (Dosé et al., 2003). A point mutation in the second conserved motif, 3THDII, eliminated the actin binding that is essential for targeting a fish myosin IIIa to filopodia tips, but not to photoreceptor calycal processes (Les Erickson et al., 2003; LinJones et al., 2004).

We used immunolabeling to show that myosin IIIa is localized around the stereocilia tips forming a thimble-like pattern. Expression of green fluorescent protein (GFP)-tagged myosin IIIa showed that the motor domain, but not the conserved tail actinbinding domain, is required for stereocilia tip localization. Furthermore, deletion of the unique $5^{\prime}$ kinase domain enhances the accumulation of myosin IIIa and results in elongation of the stereocilia and bulging of the stereocilia tips. We argue that the thimble-like 
localization and the influence myosin IIIa has on stereocilia shape reveal a previously unrecognized molecular compartment at the distal end of stereocilia, the site of actin polymerization as well as operation of the MET.

\section{Materials and Methods}

Antibodies, expression plasmids, and in situ probes. An affinity-purified polyclonal antibody (PB638) was developed in a rabbit immunized with a synthetic peptide (NPYDYRRLLRKTSQRQR) matching the C-terminus sequence of mouse myosin IIIa (Princeton Biomolecules, Langhorne, PA). Two other affinity-purified antibodies, 5525 and 5526, were generated previously (Dosé et al., 2003) in two rabbits both given injections of a peptide corresponding to the last 22 aa of the bass (Morone saxitilis) myosin IIIa C terminus. The epitope used for the bass antibody production contained extensive sequence homology to the mouse, rat, and human myosin IIIa tail end (Dosé et al., 2003). The specificity of all three myosin IIIa antibodies was tested using ectopic expression of GFPtagged plasmid DNA for the human and bass myosin IIIa in COS-7 cells (data not shown). Affinity-purified rabbit polyclonal antibodies and GFP-tagged plasmids to myosin XVa (Anderson et al., 2000) and myosin Ic (Dumont et al., 2002) were obtained from T. Friedman [National Institute on Deafness and Other Communication Disorders (NIDCD), National Institutes of Health (NIH), Bethesda, MD] and P. Gillespie (Vollum Institute, Portland, OR), respectively. Monoclonal anti- $\beta$ tubulin (clone D66) was obtained from Sigma (St. Louis, MO).

We characterized the phenotypes of cells transfected with bass and human myosin IIIa and mutations of specific domains subcloned into pEGFP-C1, -C2, -C3 (GFP) vectors (Invitrogen, Carlsbad, CA). Bar diagrams of the functional domains of GFP-tagged constructs and their abbreviations used are shown in Figure 5, $a$ and $b$. The bass constructs used were as follows: WT, wild-type bass GFP-myosin IIIa (amino acids 1-1838); MD, motor dead bass GFP-myosin IIIa (with an R-to-A mutation at amino acid position 507); $\Delta \mathrm{K}$, bass GFP-myosin IIIa $\delta$ kinase (amino acids 361-1838); $\Delta$ 3THDII, bass GFP-myosin IIIa (amino acids 1-1816). The human constructs used were as follows: WT, human GFPmyosin IIIa (amino acids $1-1615$ ); $\Delta \mathrm{K}$, human GFP-myosin IIIa $\delta$ kinase (amino acids $340-1615$ ); $\Delta 34$, human GFP-myosin IIIa $\delta$ exon 34 (deletion of exon 34, which results in missing C-terminal two-thirds of 3THD1, but 3THDII is still in frame); $\Delta \mathrm{K}-34$, human GFP-myosin IIIa $\delta$ kinase, $\delta$ exon 34 (deletion of both domains); $\Delta 33-34$, human GFPmyosin IIIa, $\delta$ exons 33 and 34 (out of frame after first five amino acids of 3 THD1 and therefore missing 3THDII as well); $\Delta \mathrm{K}-33-34$, human GFPmyosin IIIa $\delta$ kinase, $\delta$ exons 33 and 34 (deletion of all three domains).

In situ hybridization probe for myosin IIIa consisted of $1 \mathrm{~kb}$ of the myosin IIIa cDNA (AY101368; 89-1020 nucleotides) template subcloned into the pBluescript vector, using the following pair of PCR primers: 5-GAAATCATTGAAACCATCGGCAAAGG and 5-TACATCCTTCAGACTGGAAATGAGAG. The myosin XVa probe was prepared from myosin XVa plasmid (a gift from T. Friedman) as described previously (Liang et al., 1999).

Inner ear tissues. Inner ear tissues were obtained from rats, mice, guinea pigs, frogs, and chickens killed in accordance with NIH guidelines under NIDCD animal protocol 1067-02.

Immunocytochemistry. Immunolocalization of myosins was performed on whole-mount preparations of dissected organ of Corti and vestibular organs obtained from mouse, rat, guinea pig, chicken, and bullfrog. Temporal bones were gently perfused with $4 \%$ paraformaldehyde (PFA) in PBS at room temperature, through the round window, and fixed for $2 \mathrm{~h}$ as described previously (Rzadzinska et al., 2004). Organ of Corti and vestibular epithelia pieces were dissected in PBS, permeabilized with $0.5 \%$ Triton X-100 (Sigma) for $30 \mathrm{~min}$, blocked in 2-4\% bovine serum albumin, and stored overnight at $4^{\circ} \mathrm{C}$. Organ of Corti pieces were incubated with primary antibody for $2 \mathrm{~h}$ and washed three times in PBS before staining with Alexa Fluor-conjugated secondary antibody (Invitrogen) for $1 \mathrm{~h}$. Pieces were subsequently washed, counterstained with $0.001 \mathrm{U} / \mu \mathrm{l}$ Alexa Fluor 568 phalloidin (Invitrogen), washed with PBS, and mounted using Prolong anti-fade (Invitrogen). Some samples were processed for antigen retrieval. Briefly, fixed and permeabilized samples were transferred to $0.1 \mathrm{M}$ citrate buffer, $\mathrm{pH} 7.0$, and heated to $60^{\circ} \mathrm{C}$ for $30 \mathrm{~min}$ before blocking overnight and processed as before. Fluorescence confocal images were obtained with a Nikon (Tokyo, Japan) microscope using a $60 \times$ or $100 \times 1.4$ numerical aperture (NA) objective and a spinning wheel confocal unit (PerkinElmer, Wellesley, MA). We analyzed at least three samples from three animals at each age used, and representative images are shown.

Tissue preparation for electron microscopy. Freshly dissected inner ear tissues harvested from 8 -d-old rats were fixed in a mixture of $4 \% \mathrm{PFA}$ and $0.5 \%$ glutaraldehyde in $0.1 \mathrm{M}$ cacodylate buffer, $\mathrm{pH} 7.0$, with $3 \mathrm{~mm} \mathrm{CaCl}_{2}$, glycerinated, plunged into liquid Freon 22, and promptly transferred to liquid nitrogen. Frozen samples were freeze substituted in $1.5 \%$ uranyl acetate in absolute methanol at $-90^{\circ} \mathrm{C}$, infiltrated with Lowicryl HM-20 resin at $-45^{\circ} \mathrm{C}$, polymerized with UV light, thin-sectioned, and immunogold labeled as described previously (Dumont et al., 2001). Stained sections were viewed and photographed with a Leo 922 transmission electron microscope equipped with an energy filter for contrast tuning.

Cultures and transfection of rat inner ear tissue. After $\mathrm{CO}_{2}$ anesthesia, postnatal day $0(\mathrm{P} 0)$ to $\mathrm{P} 4$ rat pups were killed in accordance with $\mathrm{NIH}$ guidelines, and their temporal bones were isolated and placed in L-15 medium (Invitrogen). Pieces of organ of Corti and maculae of the utricle, saccule, and the ampullae were dissected and attached to coverslips previously coated with $150 \mu \mathrm{g} / \mu \mathrm{l}$ Cell Tak (BD Biosciences, San Jose, CA) as described previously (Rzadzinska et al., 2004). Cultures were maintained in DMEM/F-12 (Invitrogen) supplemented with 5-7\% fetal bovine serum (FBS) (Invitrogen) and $1.5 \mu \mathrm{g} / \mathrm{ml}$ ampicillin and kept at $37^{\circ} \mathrm{C}$ and $5 \% \mathrm{CO}_{2}$. Plasmid DNAs encoding the various GFP-myosin IIIa constructs were precipitated onto $1 \mu \mathrm{m}$ gold particles at $50 \mu \mathrm{g}$ of DNA to 25 $\mathrm{mg}$ of $1 \mu \mathrm{m}$ gold particles and loaded into cartridges following the Helios Gene Gun protocol (Bio-Rad, Hercules, CA). Tissue explants were transfected with these gold carriers using the Helios Gene Gun using 85-95 psi helium pressure and incubated for up to $48 \mathrm{~h}$. Samples were washed briefly with PBS, fixed at various times in 4\% PFA for at least $1 \mathrm{~h}$, and permeabilized with $0.5 \%$ Triton X-100 in PBS containing Alexa Fluor 568 phalloidin for $30 \mathrm{~min}$ before washing and mounting on a microscope slide with the anti-fade. The efficiency of transfection ranged from 0 to 3 hair cells per tissue explant, and we examined at least 20 transfected cells for each construct used.

Culture and transfection of COS-7 cells. Simian fibroblast COS-7 cells were plated on coverslips and maintained at $37^{\circ} \mathrm{C}$ in DMEM supplemented with $10 \%$ FBS. Cultures were transfected using the GeneJuice system (Novagen, San Diego, CA) according to the manufacturer's directions. Cells were fixed at various times after transfection for $20 \mathrm{~min}$ in $4 \%$ PFA in PBS, permeabilized for 10 min in $0.5 \%$ Triton X-100 in PBS, processed for immunocytochemistry or counterstained with Alexa Fluor 568 phalloidin for $20 \mathrm{~min}$, and mounted on a microscope slide with anti-fade. Live-cell imaging of transfected COS-7 cells was performed in L-15 medium. Time-lapse confocal Z-stacks were collected using a Nikon Eclipse microscope and PerkinElmer Ultraview confocal with a $100 \times 1.4$ NA objective. The extended focus rendering was performed using Volocity 3.7.0 (Improvision, Coventry, UK). We examined $>40$ cells over periods of 20 min each.

In Situ hybridization. In situ hybridization experiments were performed as described previously (Wu and Oh, 1996). Briefly, tissues were fixed in 4\% PFA in PBS overnight in the cold room and processed through graded sucrose solutions (5-30\%) for cryoprotection before cryosectioning. Digoxigenin-labeled riboprobes were produced using DNA templates as described above. The results of the in situ hybridization were then visualized using nitroblue tetrazolium/5-bromo-4chloro-3-indolyl phosphate chromatic development procedure.

\section{Results}

\section{Myosin IIIa localized to tips of developing stereocilia of inner ear hair cells}

We used three affinity-purified myosin IIIa-specific antibodies (PB638, 5525, and 5526) to characterize the steady-state distribution and temporal patterns of expression of myosin IIIa in developing and mature hair bundles from P0 through adult ( $>$ P30) 


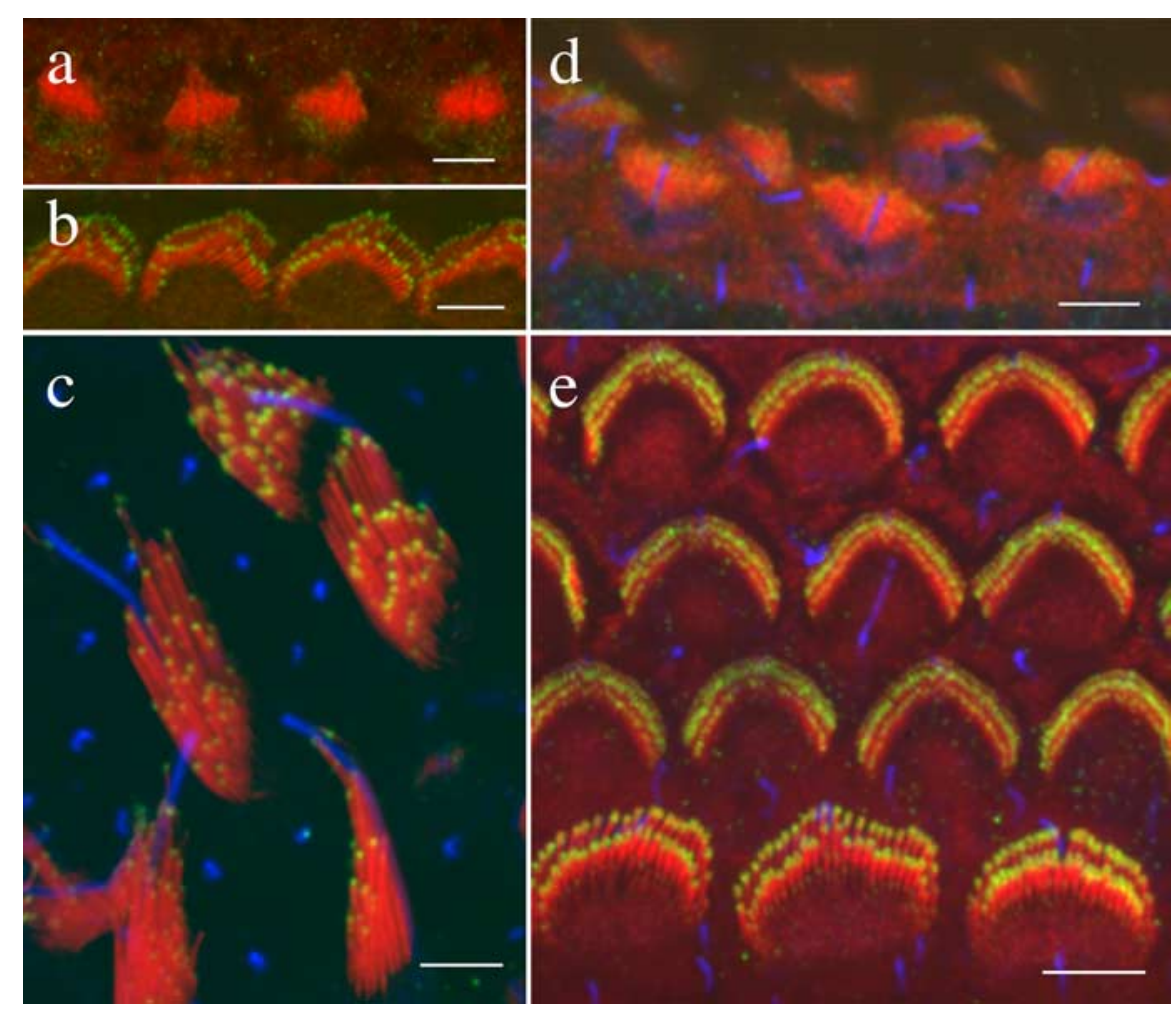

Figure 1. Myosin Illa expression lags stereocilia staircase formation. Fluorescence confocal images of whole-mount preparations of the rat organ of Corti and saccule show myosin Illa immunofluorescence (green) in the developing stereocilia bundles. Actin filaments are counterstained with rhodamine-phalloidin (red), whereas microtubules of the kinocilia are labeled with the anti-tubulin antibody (blue; $\boldsymbol{c}, \boldsymbol{e}) . \boldsymbol{a}-\boldsymbol{c}$, Myosin Illa immunoreactivity is not detectable at the stereocilia in the early stages of development in the hair cells of the apical turn of the P1 rat cochlea $(\boldsymbol{a})$ but is seen at the tips of the stereocilia of the P1 basal turn inner hair cells $(\boldsymbol{b})$ and vestibular hair cells (c) where the staircase organization is well formed. $\boldsymbol{d}, \boldsymbol{e}$, By P3, hair cells of the apical (d) and basal (e) turns of the cochlea show clearly detectable levels of myosin Illa immunoreactivity at the tips of their stereocilia (d). Scale bars, $5 \mu \mathrm{m}$

cochlea. Immunofluoresence experiments were performed using all three anti-myosin IIIa affinity-purified antibodies in rat and mouse inner ear sensory epithelia. The overall results were indistinguishable regardless of the antibodies and rodent species used, and we show in the figures representative results using PB638 in rat preparations. In the basal turns of the rat cochlea, stereocilia emerge from the apical surface of the inner and outer hair cells at embryonic day 18, and by P21, the hair bundles have reached their full, mature length and lost their kinocilium (Tilney et al., 1988; Kaltenbach et al., 1994; Zine and Romand, 1996). Maturation of inner hair cells of the basal region precedes the maturation of outer hair cells, and development proceeds toward the apex of the cochlea over a few days (Zine and Romand, 1996). In the comparatively immature outer hair cells of the apical regions of the P0 rat cochlea, no myosin IIIa immunolocalization could be detected at the tips of the emerging stereocilia (Fig. 1a). In the longer stereocilia of the inner hair cells of the basal turn of the P0 cochlea and in the vestibular hair cells where the hair bundle already has the characteristic staircase shape, we observed distinct myosin IIIa immunolabeling at the tips of stereocilia (Fig. 1b,c). By P3, myosin IIIa labeling was observed at stereocilia tips of both inner and outer hair cells of the apical turns of the cochlea (Fig. $1 d, e)$. Myosin IIIa immunolabeling could not be detected in the kinocilium, which is present in all vestibular hair cells and in the immature cochlear hair cells (Fig. $1 c-e$ ).
Myosin IIIa expression increased as stereocilia elongated in the cochlea We observed the highest levels of myosin IIIa immunoreactivity at tips of stereocilia between P6 (Fig. 2a,b) and P10 (Fig. 2c,d) in the rat cochlea. Because stereocilia range between 200 and $500 \mathrm{~nm}$ in diameter, the fluorescence images are diffraction limited. However, in the longest and widest stereocilia of the cochlea (from the inner hair cells of the apical turn), the tips exhibited a clearly distinguished thimblelike pattern of fluorescence. Confocal images of side views (Fig. $2 a, d$ ) or of top views (Fig. $2 e-g$ ) of the tallest row of stereocilia showed a ring pattern of labeling surrounding the unlabeled stereocilia actin core. The staining pattern for the middle and short rows of stereocilia often showed an asymmetric pattern with labeling more concentrated toward the tallest row of stereocilia (Fig. $2 d$, arrows). The pointed fluorescence pattern was consistent with the asymmetric pointed shape of the second row of stereocilia as seen by electron microscopy (Kachar et al., 2000). In the outer hair cells, the middle-row stereocilia often showed an apparent higher level of myosin IIIa immunoreactivity (Figs. $1 e, 2 b$ ).

\section{Changes in myosin IIIa}

immunoreactivity during late stages of stereocilia maturation

Using our standard protocol for immunofluorescence, we initially observed that myosin IIIa immunofluorescence levels dropped from the peak of expression between P6 and P10 (Fig. $2 a-d$ ) to very low levels in the adult cochlea (Fig. $3 a-f$ ), whereas the thimble-like pattern of fluorescence appeared more restricted to the stereocilia tip (compare Figs. $2 a, 3 a, g$ ). In the hair cells of the basal turn of adult guinea pig cochlea, myosin IIIa staining was barely detectable at the stereocilia tips (Fig. 3f). No labeling was detected in the rat or mouse adult hair cell stereocilia (Fig. $3 d, e$ ). In vestibular hair cells, myosin IIIa was localized at the tips of stereocilia and the thimble-like banding pattern could be recognized up to P20 (Fig. 3j). In stereocilia of adult vestibular hair cells, the fluorescence intensity of the labeling decreased and appeared as a series of puncta (Fig. $3 k$ ).

The progressive loss of immunofluorescence intensity at the later stage of maturation of the stereocilia observed in our immunofluorescence assay was inconsistent with the reported involvement of MYO3A in late-onset deafness (Walsh et al., 2002). We explored the possibility that the loss of immunoreactivity might be caused by some form of epitope masking. We used an antigen retrieval step, which consisted of incubation of the fixed tissue for $30 \mathrm{~min}$ at $60^{\circ} \mathrm{C}$ in citrate buffer, and observed a robust recovery of the immunolabeling signal intensity in the stereocilia of all adult hair cells, including adult rat, mouse, and guinea pig cochlear (Fig. 3g-i) and rat vestibular (Fig. $3 l$ ) hair cells. With this antigen retrieval protocol (data not shown), we also confirmed the progressive increase of immunofluorescence intensity and labeling pattern observed during the early developmental stages with our 
standard protocol and the late confinement or restriction of the thimble-like fluorescence within the stereocilia tips.

We sought to independently confirm the myosin IIIa expression throughout hair cell development and maturation using in situ hybridization. We hybridized mouse cryosections of the cochlea and ampullae from P0, P8, P15, P30, and P50 with a $1 \mathrm{~kb}$ riboprobe directed to the mouse myosin IIIa $\mathrm{N}$ terminus and used a $1 \mathrm{~kb}$ myosin XVa probe as a hair cell marker (Liang et al., 1999). We observed a strong hybridization signal from inner and outer hair cells at P8 (supplemental Fig. 1a, available at www.jneurosci.org as supplemental material) as well as from hair cells of the ampullae (supplemental Fig. 1b, available at www.jneurosci.org as supplemental material). Moreover, clear hybridization labeling in the P50 mouse cochlear and vestibular hair cell (supplemental Fig. $1 c, d$, available at www.jneurosci.org as supplemental material) was observed. Positive hybridization experiments in the adult cochlea suggested that myosin IIIa is expressed in adult hair cells, consistent with our immunocytochemical observations.

We extended our findings on myosin IIIa distribution by studying bullfrog hair cells with our standard protocol and with the added antigen retrieval step. The thimble-like distribution of myosin IIIa in stereocilia was observed under either protocol in this non-mammalian vertebrate (Fig. 4). In the frog saccule and utricle hair cells, the myosin IIIa immunofluorescence of the middle-row stereocilia exhibited a pointed pattern (Fig. $4 a$, arrow, $b$ ). In the very long stereocilia of the ampullae hair cells, the myosin IIIa immunofluorescence extended from the tips downward along the stereocilia shaft in a comet-like pattern (Fig. $4 d-f$ ). Some of the longest stereocilia of the ampullae exhibited slightly bulbous or club-shaped tips, and these unusual phenotypes were also strongly positive for myosin IIIa labeling, especially over the enlarged region of the tips (Fig. $4 c-f$, arrowheads). We observed these various degrees of bulging of the tips in $\sim 1-2 \%$ of the cells

We also evaluated myosin IIIa localization by postembedding immunogold electron microscopy of rat (supplemental Fig. $2 a-c$, available at www.jneurosci.org as supplemental material) and chick (supplemental Fig. $2 d$, available at www.jneurosci.org as supplemental material) hair cells. Labeling could be observed around the stereocilia tips in longitudinal and perpendicular sections in a pattern consistent with the thimble-like staining pattern of the immunofluorescence confocal images.

\section{GFP-myosin IIIa was targeted to the tips of stereocilia in transfected hair cells}

To assess the ability of hair cells to target and properly compartmentalize exogenous myosin IIIa to the tips of stereocilia, we transfected hair cells of $\mathrm{P} 4-\mathrm{P} 11$ rat inner ear sensory epithelia cultures with bass (Fig. 5a) or human (Fig. 5b) GFP-tagged myosin IIIa. Within 18-48 h after hair cell transfection, myosin IIIa was concentrated at the tip of each stereocilium in transfected cells of organ of Corti and vestibular epithelia (Fig. $5 c-f$ ). Highmagnification of the well resolved stereocilia images suggest that GFP-myosin IIIa forms a thimble-like pattern at the stereocilia tips (Fig. $5 d, f$, arrowheads) similar to that observed by myosin IIIa immunolocalization. We compared transfected hair cells from the organ of Corti with nontransfected neighboring cells to assess the effect of GFP-myosin IIIa overexpression on promoting actin elongation and stereocilia lengthening. Unlike our observations with GFP-myosin XVa (H. W. Lin and B. Kachar, unpublished data), GFP-myosin IIIa overexpression did not produce evident changes in stereocilia length (data not shown).

\section{Targeting and structural effects of mutated myosin IIIa in stereocilia}

Previous studies on myosin IIIa have identified critical domains in which mutations affected the localization pattern of heterologously expressed GFP-myosin IIIa in filopodia of cultured cell 

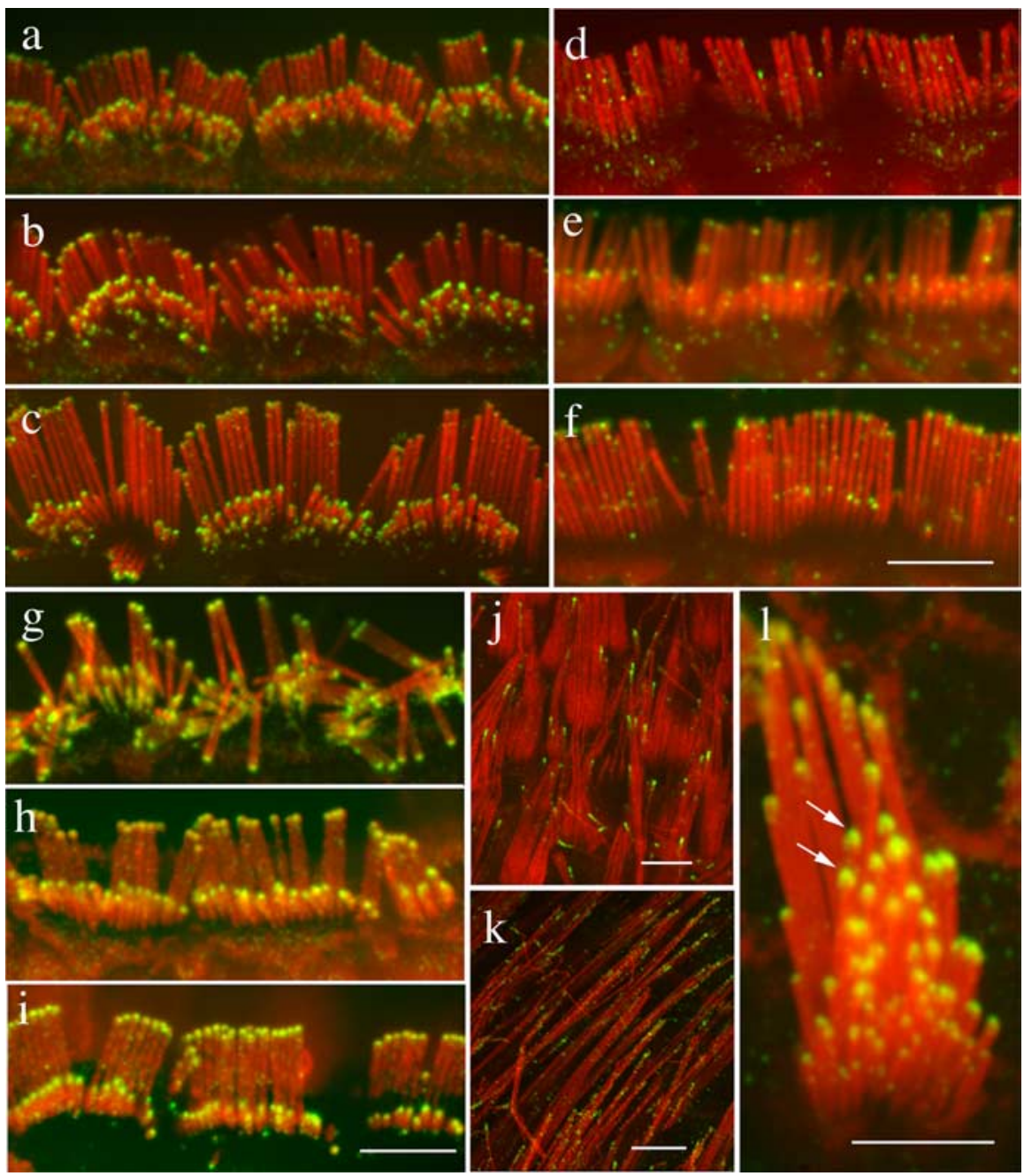

Figure 3. Myosin Illa epitopes are masked as stereocilia mature. The immunoreactivity to myosin Illa (green) changes at the late stages of stereocilia development (counterstained for actin in red). $\boldsymbol{a}-\boldsymbol{c}$, Inner hair cells from the apical turn of the (rat) cochlea from P16 (a), P18 (b), and P24 (c) showing decreased immunofluorescence and condensation into the stereocilia tip.d, $\boldsymbol{f}$, Fluorescence is absent in the tips of stereocilia of inner hair cells of the basal turn of the adult rat $(\boldsymbol{d})$ and mouse $(\boldsymbol{e})$ cochlea and barely detectable in the adult guinea pig $(\boldsymbol{f}) . \boldsymbol{g}-\boldsymbol{i}$, After antigen retrieval, myosin Illa immunofluorescence is observed at the tips of stereocilia in the inner hair cells of the basal turn of the adult rat $(\boldsymbol{g})$, mouse $(\boldsymbol{h})$, and guinea pig $(\boldsymbol{i})$ cochlea. $\boldsymbol{j}-\boldsymbol{I}$, Myosin Illa immunofluorescence at the tips of stereocilia of P20 $(\boldsymbol{j})$ and adult $(\boldsymbol{k}, \boldsymbol{I})$ rat vestibular hair cells without $(\boldsymbol{j}, \boldsymbol{k})$ and with $(\boldsymbol{I})$ the antigen retrieval step. Scale bars: $\boldsymbol{a}-\boldsymbol{i}, \boldsymbol{I}, 5 \mu \mathrm{m} ; \boldsymbol{j}, \boldsymbol{k}, 2 \mu \mathrm{m}$.

lines and rod retinal cells (Dosé et al., 2003; Les Erickson et al., 2003; Lin-Jones et al., 2004). We analyzed GFP-myosin IIIa localization in cultured rat organ of Corti and vestibular hair cells (Fig. 5) and examined stereocilia phenotypes resulting from transfection of bass (Fig. 5a) and human (Fig. 5b) myosin IIIa mutants. Myosin IIIa accumulation in the tips of stereocilia depended on the presence of a functional motor, because the GFPmyosin IIIa lacking a functional motor (MD construct) failed to target the stereocilia and instead accumulated in the cytoplasm (Fig. $5 g$ ).

The class III myosin-specific N-terminal kinase motif appears to have an important role in myosin IIIa function. In hair cells transfected with human or bass GFP-myosin IIIa lacking the kinase domain $\Delta \mathrm{K}$, the protein accumulated at higher amounts at the tips of stereocilia and often produced elongation of the stereocilia and bulging of the tip as well as an apparent floppiness of the normally rigid stereocilia (Fig. $5 h-j$ ). Both the elongation and bulging were evident after visual inspection of the fluorescence images (in the $>40$ transfected cells observed) but were difficult to quantify because the entire stereocilia bundle staircase organization was often disrupted (Fig. $5 h-j$ and supplemental Fig. $3 a, c$, available at www.jneurosci. org as supplemental material). Interestingly, the $\Delta \mathrm{K}$-transfected nonsensory cells of either the organ of Corti or vestibular sensory epithelia, which commonly have short microvilli on their surface, exhibited unusually long and irregular actin protrusions (supplemental Fig. 3b, available at www.jneurosci.org as supplemental material). These long actin protrusions were not observed when these cells were transfected with the full-length GFP-myosin IIIa (supplemental Fig. 3b, inset, available at www.jneurosci.org as supplemental material).

A conserved tail domain motif, 3THDII, has been shown previously to bind actin and was required for localization of GFP-myosin IIIa to filopodia tips in a heterologous expression system (Les Erickson et al., 2003). We tested whether elimination of the 3THDII region from either the bass or human transcripts affected the targeting of myosin IIIa and influenced the shape of the stereocilia. We tested two mutant variants of myosin IIIa associated with the 3THD domains. The human GFP-myosin IIIa $\Delta 33-34$ lacks exons 33 and 34, which removes all but the first five amino acids of 3THDI and also creates a frame shift altering the rest of the tail, thereby eliminating 3THDII. The myosin IIIa $\Delta 34$ variant removes the second two-thirds of 3THDI, because it does not cause a frame shift, so 3THDII is still present at the C terminus. The $\Delta 34$ is a naturally occurring splice variant seen in mouse (Walsh et al., 2002), and the $\Delta 33-34$ variant was identified among other variants in human cDNA (A. C. Dosé, unpublished results). Both of the human GFP-myosin IIIa tail variants efficiently localized to stereocilia tips and showed a phenotype indistinguishable from the wild-type myosin IIIa overexpression (Fig. $5 k, l$ ). A species difference between bass and human was observed in the localization of tail mutants in hair cells. Bass GFP-myosin IIIa missing exclusively the final 22 amino acids (3THDII) resulted in an attenuated expression in stereocilia tips (data not shown).

Because the absence of the kinase domain affected the functional activity of myosin IIIa, but absence of 3THDII did not (unlike in cultured cell lines), we sought to examine the effect of mutations in both domains simultaneously. Expressed myosin IIIa lacking both the kinase domain and residues encoded by exons 33 and $34(\Delta \mathrm{K}-33-34)$ no longer exhibited the aberrant $\Delta \mathrm{K}$ phenotype and was indistinguishable from the wild type (Fig. $5 \mathrm{~m}$.) However, expression of myosin IIIa lacking the kinase domain and only exon $34(\Delta \mathrm{K}-34)$, hence with an in-frame 3THDII, caused increased fluorescence, stereocilia elongation, and bulging at the tips (Fig. $5 n$ and supplemental Fig. $3 c$, available at www.jneurosci.org as supplemental material), resembling the $\Delta \mathrm{K}$ 
phenotype. Similarly, supporting cells expressing $\Delta \mathrm{K}-34$ showed elongated and irregular microvilli with GFP fluorescence at their tips. These data suggest that the presence of 3THDII is required for the $\Delta \mathrm{K}$ phenotype.

\section{Targeting and effect of mutants of} myosin IIIa in COS-7 cells

We also evaluated the distribution and effects of GFP-myosin IIIa and the various myosin IIIa mutants on filopodia in transfected COS-7 cells (Fig. 6). Under our culture conditions, COS-7 cells exhibited very few filopodia. When COS-7 cells were transfected with GFP-myosin IIIa, the fluorescence signal was observed at the tips and along the very few filopodia even after $60 \mathrm{~h}$ of transfection (Fig. $6 a$, arrows). On the other hand, GFP-myosin IIIa $\Delta \mathrm{K}$ caused a progressive increase in the number of filopodia that could be clearly visualized in all transfected cells after $24 \mathrm{~h}$ (Fig. $6 b$ ) and $48 \mathrm{~h}$ (Fig. 6c). Myosin IIIa mutants $\mathrm{MD}, \Delta 34, \Delta 33-34$, and $\Delta \mathrm{K}-33-34$ all failed to localize to filopodia (Fig. $6 d-g$ ). When prepared as a construct with just the $3 \mathrm{TH}$ DII region but missing most of 3THDI $(\Delta \mathrm{K}-34)$, the kinase mutation increased the number of myosin IIIa-positive filopodia in COS-7 cells (Fig. 6h, arrows). The number of filopodia seen in $\Delta \mathrm{K}-34$ transfected cells was less than that induced by the kinase domain deletion $(\Delta \mathrm{K})$ alone (Fig. 6, compare $h, i$ ). Myosin IIIa $\Delta \mathrm{K}$ accumulated at the distal ends of filopodia, and often the fluorescence extended toward the base of the filopodia, forming a comet-like pattern (Fig. 6i, arrowhead). We examined the dynamics of GFP-myosin IIIa $\Delta \mathrm{K}$ fluorescence in filopodia of COS-7 cells in time-lapse video recordings after $24 \mathrm{~h}$ after transfection. Surprisingly, although the numerous filopodia exhibited active pivoting motions about their base (supplemental movie 1, available at www.jneurosci.org as supplemental material) and intrafilopodial movements of GFP-myosin IIIa fluorescence puncta (supplemental movie 2, available at www. jneurosci.org as supplemental material), filopodial extension and retraction (Faix and Rottner, 2006) were rarely detected within the 20 min time-lapse recordings.

\section{Myosin IIIa localization in stereocilia is distinct from myosin Ic and} myosin XVa

To compare the localization of myosin IIIa with other stereocilia myosins reported to be present at the stereocilia tip, we examined in comparable experiments the precise compartmentalization of three unconventional
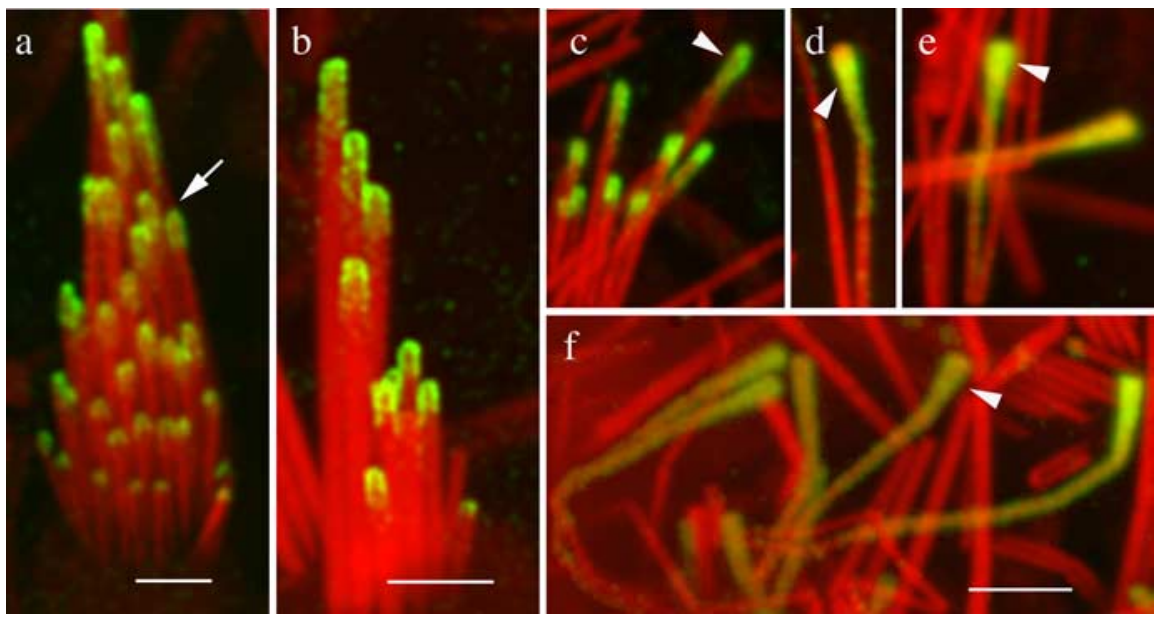

Figure 4. Myosin IIla localization at regular and club-shaped stereocilia tips in the bullfrog inner ear. Immunofluorescence confocal images of utricle $(\boldsymbol{a}, \boldsymbol{b})$ and ampullae $(\boldsymbol{c}-\boldsymbol{f})$ show myosin Illa immunoreactivity around the tips of stereocilia of adult frog vestibular hair cells. $\boldsymbol{a}$, The arrow shows the thimble-like and pointed shape of the labeling in some middle-row stereocilia. $\boldsymbol{b}$, The same pattern of labeling is observed after antigen retrieval. $\mathbf{c}-\boldsymbol{f}$, Some of the very long stereocilia of the ampullae show higher levels of immunoreactivity and bulged or club-shaped tips. In some stereocilia $(\boldsymbol{d}-\boldsymbol{f})$, the immunofluorescence may extend down the shaft for several micrometers. Scale bars, $2 \mu \mathrm{m}$.

$\mathbf{a}$
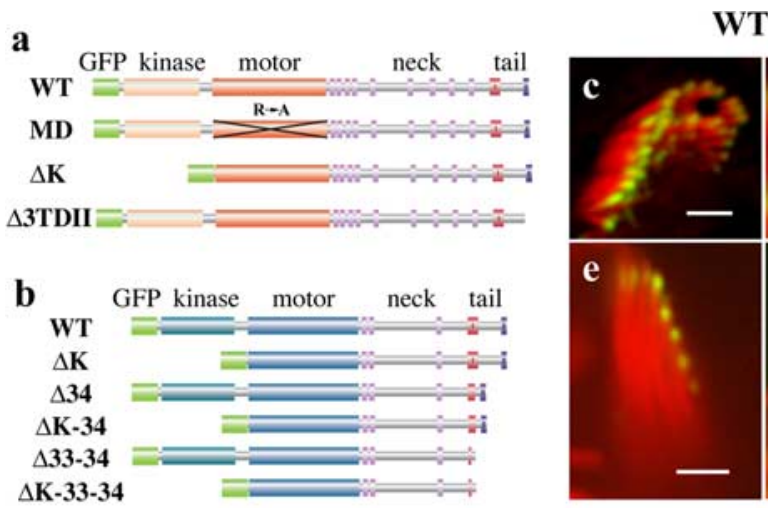

WT

MD

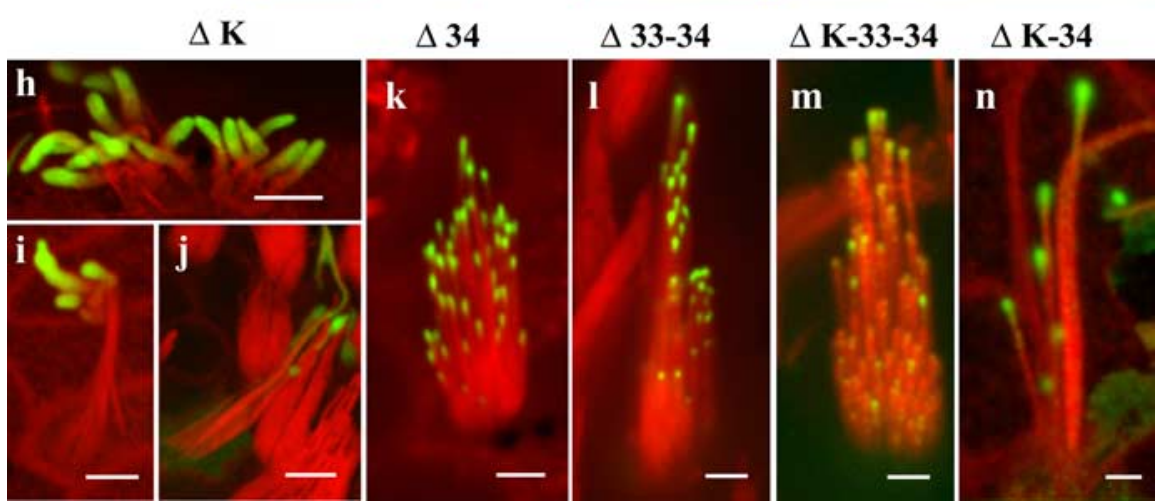

Figure 5. Overexpression of myosin IIla functional mutants in hair cells. $\boldsymbol{a}, \boldsymbol{b}$, Schematic representation of bass ( $\boldsymbol{a}$ ) and human (b) GFP-myosin Illa mutants. c-n, Confocal images of cultured organ of Corti and vestibular hair cells transfected for $18-24 \mathrm{~h}$ with GFP-myosin Illa mutants (green) and counterstained for actin with phalloidin (red). $\boldsymbol{c}-\boldsymbol{f}$, Human $(\boldsymbol{c}, \boldsymbol{d})$ or bass $(\boldsymbol{d}, \boldsymbol{f})$ wild-type GFP-myosin Illa localizes at the tips of stereocilia of transfected inner hair cells $(\boldsymbol{c}, \boldsymbol{d})$ or vestibular hair cells $(\boldsymbol{e}, \boldsymbol{f})$. $\boldsymbol{g}$, Inactivation of the bass myosin IIla motor domain causes GFP-myosin IIla to accumulate in the cytoplasm and confirms that stereocilia tip localization requires a functional motor domain. $\boldsymbol{h}-\boldsymbol{j}$, Images of accumulation of fluorescence and extension and bulging of the tips of stereocilia in auditory $(\boldsymbol{h})$ and vestibular $(\boldsymbol{i}, \boldsymbol{j})$ hair cells transfected with either bass GFP-myosin IIla $\Delta \mathrm{K}(\boldsymbol{h})$ or human GFP-myosin IIla $\Delta K(\boldsymbol{i}, \boldsymbol{j}) \cdot \boldsymbol{k}, \boldsymbol{I}$, The deletion mutants GFP-myosin Illa $\Delta 34(\boldsymbol{k})$ and GFP-myosin IIla $\Delta 33-34(\boldsymbol{I})$ do not affect localization or the extent of accumulation in stereocilia tips. $\boldsymbol{m}, \boldsymbol{n}$, The kinase domain mutation increases the expression of myosin Illa containing deletions of exon $34(\Delta K-34)(\boldsymbol{n})$ but not of a larger region of the conserved tail $(\Delta \mathrm{K}-33-34)(\boldsymbol{m})$. Stereocilia of hair cells with $\Delta K-34$ mutations elongate and have bulbous tips. Scale bars, $2 \mu \mathrm{m}$. MD, Inactive motor domain; $\Delta K$, kinase deletion; $\Delta 34$ (human), lacking exon 34, but with 3THDIl intact; $\Delta 3$ TDIl (bass) and $\Delta 33-34$ (human) conserved actinbinding domain 3THDII deletion or out of frame; $\Delta \mathrm{K}-34$ and $\Delta \mathrm{K}-33-34$ are combinations of deletions removing the kinase domain with deletions in the tail domain; WT, wild type. 

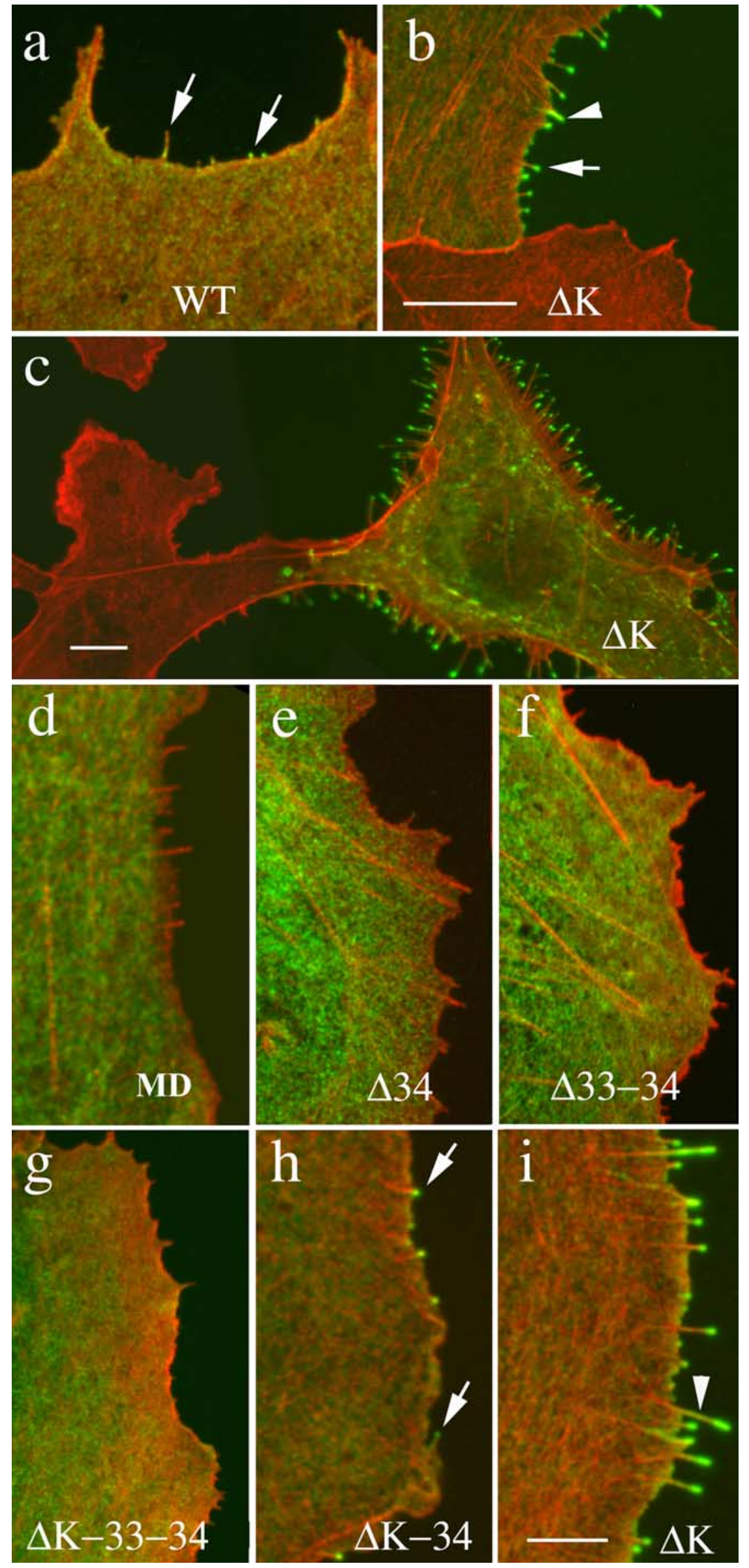

myosins with immunofluorescence and exogenous gene transfection. We confirmed that myosin XVa immunofluorescence (Fig. 7a) was matched by the localization of GFPmyosin XVa (Fig. 7b) into a cap-like structure at the very tips of stereocilia (Rzadzinska et al., 2004). In contrast, myosin Ic was expressed in a separate and distinct compartment from myosin IIIa or XVa. Myosin Ic immunofluorescence was distributed along the entire length of the stereocilia shafts and excluded from a cap region at the tips of stereocilia (Fig. $7 c$, arrowheads). Overexpression of myosin Ic confirmed that myosin Ic is distributed along the entire length of the stereocilia and excluded from the stereocilia cap (Fig. $7 d$, arrowheads). The immunofluorescence and localization of GFP-tagged myosins XVa and Ic clearly contrasts with the localization of myosin IIIa as shown in comparable close-up views (Fig. 7e,f).

\section{Discussion}

The unique pattern of localization of myosin IIIa in stereocilia

We show that myosin IIIa forms a thimble-like pattern at stereocilia tips. In electron micrographs, the tips of stereocilia show an electron-dense structure called tip density (Kachar et al., 2000). The tip density overlays the actin core and underlies the stereocilia membrane at the lower insertion site of the tip link. In contrast to previous reports on the distribution of myosin Ic (Garcia et al., 1998; Steyger et al., 1998), we show that it is excluded from the tip density region and distributed along the stereocilia shaft. Although myosin XVa forms a cap-like pattern at the top of the actin core matching the tip density, myosin IIIa forms a thimble-like pattern (Fig. 7e) that encircles the tip density region and extends further down the shaft of the stereocilia. Our results suggest that

\section{$\leftarrow$}

Figure 6. Targeting and structural effects of mutated myosin IIla in COS-7 cells. COS-7 cells were transfected for 24 - $48 \mathrm{~h}$ with GFP-tagged myosin Illa constructs (green) described in Figure 5 and counterstained for actin with phalloi$\operatorname{din}$ (red). $\boldsymbol{a}$, Wild-type GFP-myosin Illa (green) is localized at the tip and along the rare filopodia (arrows). $\boldsymbol{b}, \boldsymbol{c}$, Cells transfected with $\Delta \mathrm{K}$ for $24 \mathrm{~h}(\boldsymbol{b})$ and $48 \mathrm{~h}(\boldsymbol{c})$ show progressive accumulation of filopodia with strong fluorescence at their tips $(\boldsymbol{b}$, arrow). $\boldsymbol{b}, \boldsymbol{i}$, Fluorescence frequently extends down the length of the filopodia (arrowheads). $\boldsymbol{d}-\boldsymbol{g}$, Mutations in the myosin motor domain $(M D ; \boldsymbol{d})$, the 3DTHII domain $(\Delta 34$; $\boldsymbol{e})$, both conserved domains $(\Delta 33-34 ; \boldsymbol{f})$, or the combination of deletion of the kinase domain and the tail region ( $\Delta K-33-$ $34 ; \boldsymbol{g})$ have few filopodia, and the myosin does not concentrate in the filopodia tips. $\boldsymbol{h}$, Moderate numbers of filopodia positive for myosin Illa at their tips are observed with the $\Delta \mathrm{K}-34$ mutation (compare $\boldsymbol{h}, \boldsymbol{i}$ ). Scale bars: $\boldsymbol{a}, \boldsymbol{b}, \boldsymbol{d}-\boldsymbol{i}, 1 \mu \mathrm{m}$; c, $2 \mu \mathrm{m}$. 
these three myosins have an overall distinct pattern of distribution, but, at the same time, there is some overlap of localization around the stereocilia tip (illustrated in supplemental Fig. 4, available at www.jneurosci.org as supplemental material). The thimble-like pattern of localization of myosin IIIa at stereocilia tips suggests an additional "compartment" to the structurally defined actin core and tip density. However, we cannot rule out that the myosin IIIa molecules overlaying the paracrystalline actin core are part of the tip density. The potential roles of myosin IIIa at stereocilia tips are considered.

\section{Role of myosin IIIa in maintenance of stereocilia shape}

Although stereocilia, microvilli, and filopodia share numerous features, one key difference pertains to the stability of each protrusion. Microvilli and filopodia are formed and degraded with specific halflives, whereas stereocilia are formed once in development, continually renewed, and last a lifetime barring an injury. Over time, stereocilia are maintained by continuous turnover, and their steady-state length and tip shape are dynamically maintained (for review, see Lin et al., 2005). Stereocilia start their elongation process before detectable amounts of the myosin IIIa are present at their tips (Fig. 1a). The progressive accumulation and subsequent condensation of myosin IIIa at the tips could contribute to stabilization of the stereocilia length and to shaping the stereocilia tip. An indication that myosin IIIa at the stereocilia tip can influence its shape comes from the result of overexpression of the kinase deletion mutants that, after accumulation of the protein at the tips, produce elongation of the stereocilia, loss of apparent rigidity, and bulging of the tip. Interestingly, high levels of myosin IIIa correlates with bulging of the tips that are occasionally observed in club-shape stereocilia in the ampullae of the frog (Fig. $4 c-f$ ). Similar effects were seen in filopodia in cultured COS-7 (Fig. 6) and HeLa (Les Erickson et al., 2003) cell lines, calycal processes in Xenopus photoreceptors (Lin-Jones et al., 2004), and stereocilia (Fig. 5). One is tempted to hypothesize that myosin IIIa has a role in the actin bundle maintenance and stability and that the kinasenegative mutant is a hypermorphic mutation that exaggerates the myosin IIIa function.

\section{How does myosin IIIa reach the tips of stereocilia and how is the process regulated?}

Mutation in the myosin IIIa motor domain prevented the protein from reaching the stereocilia tips. Because actin filaments in stereocilia are uniformly polarized with their barbed end pointing to the tips, one can infer that myosin IIIa is a barbed end-directed motor and that the targeting of myosin IIIa to the tips of stereocilia depends on a motor-driven translocation along the actin filaments. The lack of the functional motor domain also prevents myosin IIIa from localizing properly in Xenopus photoreceptors (Lin-Jones et al., 2004). Interestingly, the loss of the tail actin binding site, 3THDII, prevents the myosin IIIa from localizing to the tips of filopodia as was also observed for HeLa cells (Les Erickson et al., 2003). However, in hair cells, a lack of 3THDII did not prevent accumulation of myosin IIIa at the tips of stereocilia. Similarly, myosin IIIa missing 3THDII localizes normally in $\mathrm{Xe}$ nopus photoreceptors (Lin-Jones et al., 2004). It is possible that in these cell types the endogenous myosin IIIa is assisting in the proper localization of the mutant myosin either by a cargomediated interaction or even forming heterologous dimers. A myosin IIIa phenotype associated with loss of the kinase domain is more universal. In COS-7 cells, the localization to filopodial tips is more pronounced because it was also previously shown for HeLa cells (Les Erickson et al., 2003). In both hair cells and photoreceptors, lack of the kinase domain results in an apparent upregulation of activity. Interestingly, this upregulation also re- 
quires 3THDII, because in hair cells, the $\Delta \mathrm{K}-33-34$ (lacking 3THDII) resembles the wild type and not the hypermorphic $\Delta \mathrm{K}$ phenotype. It appears that the regulation is complex and will require more detailed mutational analysis to identify the combined role of the kinase and tail domain.

\section{Other possible functions for myosin IIIa in stereocilia}

Although some stereocilia membrane proteins can diffuse laterally to distribute along the stereocilia membrane (Grati et al., 2006), other proteins including components of the tip link complex may require active transport. Myosin XVa has been shown to shuttle the PDZ protein whirlin in stereocilia and to undergo intrafilopodial motility in transfected cultured cells (Belyantseva et al., 2005). Myosin X, an unconventional myosin similar to myosin XVa and IIIa that localizes to the tip of filopodia, was also shown to exhibit intrafilopodial motility and could shuttle filopodia components toward the tips (Berg and Cheney, 2002; for review, see Sousa and Cheney, 2005). Myosin IIIa is also in a position to shuttle cargo to the stereocilia tip. Intrafilopodial movements of GFP-myosin IIIa can be seen in COS-7 cells. Similarly, in Drosophila photoreceptors, NINAC (myosin IIIa) interacts with the adapter protein INAD, which coordinates a signaling complex via its multiple PDZ domains. INAD binds directly to rhodopsin, phospholipase $\mathrm{C}$, protein kinase $\mathrm{C}$, and the lightsensitive channel subunits TRP and TRPL, necessary for visual transduction (Cronin et al., 2004; Lee and Montell, 2004; Li and Montell, 2000). A mammalian INAD (Philipp and Flockerzi, 1997) was identified by reverse transcription-PCR in the cochlea (Walsh et al., 2002), and the possibility exists that myosin IIIa contributes to transport and assembly of the tip link complex, including a TRP family member that has been postulated as a likely candidate for the MET channel (for review, see Gillespie et al., 2005).

Another possibility is that myosin IIIa contributes to MET adaptation

Current models of MET adaptation suggest that myosin Ic present at either or both ends of the tip link would mediate tension on the MET gating element (Stauffer et al., 2005). Our data suggest that myosin Ic is localized along the stereocilia length but not at stereocilia tips, the site of the lower insertion of the tip link. If myosin Ic is concentrated into an adaptation complex at the upper insertion site of the tip link, then we are unable to visualize this complex or it is indistinguishable from nonadaptation myosin Ic complexes along the stereocilia (Fig. 7c). Additional myosins may play a role in MET adaptation (Kros et al., 2002). Our data do not show localization of myosin IIIa at the upper insertion site of the tip link. However, myosin IIIa is present at the lower insertion site. The higher level of immunoreactivity for myosin IIIa and the pointed shape of the labeling observed for the middle rows of stereocilia match the shape of the tip link lower insertion site at the tips of these stereocilia (Kachar et al., 2000). By forming a ring around the tip of stereocilia, myosin IIIa could also adjust tension or "tenting" of the membrane (Kachar et al., 2000) and indirectly influence tip link tension or channel gating.

\section{What is the underlying mechanism for the late-onset deafness caused by myosin IIIa mutations?}

Mutations of human MYO3A produce late-onset deafness (DFNB30) commencing at the second decade (Walsh et al., 2002), well after the onset of full hearing and after the period of stereocilia elongation. The spatial and temporal patterns of myosin IIIa localization at stereocilia tips and the effects of deletion mutants of myosin IIIa on stereocilia and other actin protrusions suggest the physiopathology of DFNB30 is likely to involve disruption of stereocilia structure and function. We cannot explain why DFNB30 patients with recessive null mutations of myosin IIIa have normal vestibular function and the hearing loss only appears decades after birth. Perhaps myosin IIIb, a short isoform of myosin III lacking 3THDII, can compensate for myosin IIIa (Dosé and Burnside, 2002). Our results showing that mutations of the distal region of the myosin IIIa tails did not reduce its ability to localize at stereocilia tips suggest that myosin IIIb may well compensate for loss of function of myosin IIIa. The localization and expression levels of myosin IIIb and its ability to compensate for myosin IIIa need to be determined to elucidate why the recessive null mutations of myosin IIIa cause hearing loss only decades after birth and do not affect vestibular function.

\section{References}

Anderson DW, Probst FJ, Belyantseva IA, Fridell RA, Beyer L, Martin DM, Wu D, Kachar B, Friedman TB, Raphael Y, Camper SA (2000) The motor and tail regions of myosin XV are critical for normal structure and function of auditory and vestibular hair cells. Hum Mol Genet 9:1729-1738.

Belyantseva IA, Boger ET, Naz S, Frolenkov Naz, Sellers JR, Ahmed ZM, Griffith AJ, Friedman TB (2005) Myosin-XVa is required for tip localization of whirlin and differential elongation of hair-cell stereocilia. Nat Cell Biol 7:148-156.

Berg JS, Cheney RE (2002) Myosin-X is an unconventional myosin that undergoes intrafilopodial motility. Nat Cell Biol 4:246-250.

Boeda B, El-Amraoui A, Bahloul A, Goodyear R, Daviet L, Blanchard S, Perfettini I, Fath KR, Shorte S, Reiners J, Houdusse A, Legrain P, Wolfrum U, Richardson G, Petit C (2002) Myosin VIIa, harmonin and cadherin 23 , three Usher I gene products that cooperate to shape the sensory hair cell bundle. EMBO J 21:6689-6699.

Cronin MA, Diao F, Tsunoda S (2004) Light-dependent subcellular translocation of Gqalpha in Drosophila photoreceptors is facilitated by the photoreceptor-specific myosin III NINAC. J Cell Sci 117:4797-4806.

Dosé AC, Burnside B (2000) Cloning and chromosomal localization of a human class III myosin. Genomics 67:333-342.

Dosé AC, Burnside B (2002) A class III myosin expressed in the retina is a potential candidate for Bardet-Biedl syndrome. Genomics 79:621-624.

Dosé AC, Hillman DW, Wong C, Sohlberg L, Lin-Jones J, Burnside B (2003) Myo3A, one of two class III myosin genes expressed in vertebrate retina, is localized to the calycal processes of rod and cone photoreceptors and is expressed in the sacculus. Mol Biol Cell 14:1058-1073.

Dumont RA, Zhao YD, Holt JR, Bahler M, Gillespie PG (2002) Myosin-I isozymes in neonatal rodent auditory and vestibular epithelia. J Assoc Res Otolaryngol 3:375-389.

Faix J, Rottner K (2006) The making of filopodia. Curr Opin Cell Biol $18: 18-25$

Garcia JA, Yee AG, Gillespie PG, Corey DP (1998) Localization of myosin- $\mathrm{I} \beta$ near both ends of tip links in frog saccular hair cells. J Neurosci 18:8637-8647.

Gillespie PG, Cyr JL (2004) Myosin-1c, the hair cell's adaptation motor. Annu Rev Physiol 66:521-545.

Gillespie PG, Dumont RA, Kachar B (2005) Have we found the tip link, transduction channel, and gating spring of the hair cell? Curr Opin Neurobiol 15:389-396.

Grati M, Schneider ME, Lipkow K, Strehler EE, Wenthold RJ, Kachar B (2006) Rapid turnover of stereocilia membrane proteins: evidence from the trafficking and mobility of plasma membrane $\mathrm{Ca}^{2+}$-ATPase 2 . J Neurosci 26:6386-6395.

Kachar B, Parakkal M, Kurc M, Zhao Y, Gillespie PG (2000) Highresolution structure of hair-cell tip links. Proc Natl Acad Sci USA 97:13336-13341.

Kaltenbach JA, Falzarano PR, Simpson TH (1994) Postnatal development of the hamster cochlea. II. Growth and differentiation of stereocilia bundles. J Comp Neurol 350:187-198.

Komaba S, Inoue A, Maruta S, Hosoya H, Ikebe M (2003) Determination of human myosin III as a motor protein having a protein kinase activity. J Biol Chem 278:21352-21360.

Kros CJ, Marcotti W, van Netten SM, Self van Netten, Libby RT, Brown SD, 
Richardson GP, Steel KP (2002) Reduced climbing and increased slipping adaptation in cochlear hair cells of mice with Myo7a mutations. Nat Neurosci 5:41-47.

Lee SJ, Montell C (2004) Light-dependent translocation of visual arrestin regulated by the NINAC myosin III. Neuron 43:95-103.

Les Erickson F, Corsa AC, Dosé AC, Burnside B (2003) Localization of a class III myosin to filopodia tips in transfected HeLa cells requires an actin-binding site in its tail domain. Mol Biol Cell 14:4173-4180.

Li HS, Montell C (2000) TRP and the PDZ protein, INAD, form the core complex required for retention of the signalplex in Drosophila photoreceptor cells. J Cell Biol 150:1411-1422.

Liang Y, Wang A, Belyantseva IA, Anderson DW, Probst FJ, Barber TD, Miller W, Touchman JW, Jin L, Sullivan SL, Sellers JR, Camper SA, Lloyd RV, Kachar B, Friedman TB, Fridell RA (1999) Characterization of the human and mouse unconventional myosin XV genes responsible for hereditary deafness DFNB3 and shaker 2. Genomics 61:243-258.

Lin HW, Schneider ME, Kachar B (2005) When size matters: the dynamic regulation of stereocilia lengths. Curr Opin Cell Biol 17:55-61.

Lin-Jones J, Parker E, Wu M, Dosé A, Burnside B (2004) Myosin 3A transgene expression produces abnormal actin filament bundles in transgenic Xenopus laevis rod photoreceptors. J Cell Sci 117:5825-5834.

Ng KP, Kambara T, Matsuura M, Burke M, Ikebe M (1996) Identification of myosin III as a protein kinase. Biochemistry 35:9392-9399.

Philipp S, Flockerzi V (1997) Molecular characterization of a novel human PDZ domain protein with homology to INAD from Drosophila melanogaster. FEBS Lett 413:243-248.

Porter JA, Montell C (1993) Distinct roles of the Drosophila ninaC kinase and myosin domains revealed by systematic mutagenesis. J Cell Biol 122:601-612.

Ricci AJ, Kachar B, Gale J, Van Netten SM (2006) Mechano-electrical transduction: new insights into old ideas. J Membr Biol 209:71-88.

Rzadzinska A, Schneider M, Noben-Trauth K, Bartles JR, Kachar B (2005)
Balanced levels of Espin are critical for stereociliary growth and length maintenance. Cell Motil Cytoskeleton 62:157-165.

Rzadzinska AK, Schneider ME, Davies C, Riordan GP, Kachar B (2004) An actin molecular treadmill and myosins maintain stereocilia functional architecture and self-renewal. J Cell Biol 164:887-897.

Seiler C, Ben-David O, Sidi S, Hendrich Sidi, Rusch A, Burnside B, Avraham $\mathrm{KB}$, Nicolson T (2004) Myosin VI is required for structural integrity of the apical surface of sensory hair cells in zebrafish. Dev Biol 272:328-338.

Sellers JR (2000) Myosins: a diverse superfamily. Biochim Biophys Acta 1496:3-22.

Sousa AD, Cheney RE (2005) Myosin-X: a molecular motor at the cell's fingertips. Trends Cell Biol 15:533-539.

Stauffer EA, Scarborough JD, Hirono M, Miller H, Shah K, Mercer JA, Holt JR, Gillespie PG (2005) Fast adaptation in vestibular hair cells requires myosin-1c activity. Neuron 47:541-553.

Steyger PS, Gillespie PG, Baird RA (1998) Myosin I $\beta$ is located at tip link anchors in vestibular hair bundles. J Neurosci 18:4603-4615.

Tilney LG, Tilney MS, Cotanche DA (1988) New observations on the stereocilia of hair cells of the chick cochlea. Hear Res 37:71-82.

Walsh T, Walsh V, Vreugde S, Hertzano R, Shahin H, Haika S, Lee MK, Kanaan M, King MC, Avraham KB (2002) From flies' eyes to our ears: mutations in a human class III myosin cause progressive nonsyndromic hearing loss DFNB30. Proc Natl Acad Sci USA 99:7518-7523.

Wes PD, Xu XZ, Li HS, Chien F, Doberstein SK, Montell C (1999) Termination of phototransduction requires binding of the NINAC myosin III and the PDZ protein INAD. Nat Neurosci 2:447-453.

Wu DK, Oh SH (1996) Sensory organ generation in the chick inner ear. J Neurosci 16:6454-6462.

Zine A, Romand R (1996) Development of the auditory receptors of the rat: a SEM study. Brain Res 721:49-58. 